\title{
Mechanical Design and Analysis of Hybrid Mobile Robot with Aerial and Terrain Locomotion Capability
}

\author{
Monish Koshy, ${ }^{1, *}$, Sreevishnu S. ${ }^{1}$, Anjai Krishnan ${ }^{1}$, and Gautham P. Das ${ }^{2}$ \\ ${ }^{1}$ Department of Mechanical Engineering, Amrita Vishwa Vidyapeetham, Amritapuri, India \\ ${ }^{2}$ Lincoln Centre for Autonomous Systems, School of Computer Science, University of Lincoln, United Kingdom
}

\begin{abstract}
Although different locomotion mechanisms are available, the use of only one locomotion system in a mobile robot restricts its application scenarios. Hybrid locomotion improves the maneuverability and flexibility of a robot. This paper introduces a hybrid locomotion mobile robot, a combination of quadruped and quadrotor system. The robot has a unique expediency to fly to remote places, then walk to perform close range operations in the field. The prime intention is to use the quadrotor to tackle large objects by flying over it. The four legs provide easy movements in uneven terrain. Thus, this robot can be used in erratic and dynamic environments where stability, maneuverability and flexibility are required. This system can be used as first responders in search and rescue missions, where it responds before human responders gets to the site and get the entire information of the area in detail (like spotting trapped ones, getting detailed 3D mapping etc.). This platform offers unique capabilities suited for search and rescue, disaster zone assistance and surveillances. This paper elucidates the mechanical design and analysis of a hybrid locomotion robot. The solid model of the robot was made using CATIA and further analysis like static analysis, computational fluid dynamics analysis and drop test analysis were performed in ANSYS.
\end{abstract}

Keywords- Hybrid locomotion mobile robot, Quadruped, Quadrotor

\section{Introduction}

The notion of hybrid locomotion mobile system was developed in early 1980's. Robots with single locomotion has got its own advantages and disadvantages, whereas multiple locomotion increases the maneuverability and flexibility of robot [1]. Thus, came the concept of hybrid robots, where different locomotion modes are combined together. This is to ensure that the robot is benefitted from the strengths of both locomotion modes and most of the weaknesses of the individual modes are covered by the other. Robots are able to provide useful help in disaster scenario where human intervention is difficult and dangerous. Robots are used in rescue activities because it can travel quickly to areas too dangerous for humans and can engage in the field to analyse the situation [2]. The main objective of this project is to develop a robotic platform that is suitable for search and rescue, disaster zone assistance and surveillance.

Popular terrain robots used for rescue operations include legged robots [3] and tracked robots [4, 5], as these robots have more stability in uneven surface. It is used for finding the people, who may be trapped underneath things in disaster zone and to create detailed 3D map of the site. But terrain robots are unable to surpass large obstacles and pits. Within the past decade the multi-copter aircrafts has become popular as it can be send into areas where it is too dangerous or unfeasible for human. The capability of these platforms to travel quickly to a site and to generate 3D map makes it apt for search and rescue, disaster zone assistance and surveillance. But flying robots has got its difficulty in navigating through caves and notches.

Thus, an integration of the basic aerial and terrain locomotion counterpart each other giving a better solution for locomotion challenges [6]. The goal of this work is to design a hybrid locomotion mobile robot with aerial and terrain locomotion capability. Here a combination of quadruped and quadrotor system is made. The main idea is, quadrotor thrust can be used for the stability of the system in uneven surface and it also can be used to tackle large obstacles by flying over it. The four legs make it able to walk statically stable.

This paper is organized as follows. Following the introduction, Section 2 discusses about work done in this area till now. Section 3 describes factors that influence the design. The designing of hybrid mobile robot starting with $\mathrm{CAD}$ designing and various analysis performed to optimize the design is described in Section 4. Finally, the conclusion is presented in Section 5.

\section{Related works}

The introduction of hybrid locomotion robot helped to solve various limitations and challenges faced by individual locomotion mechanisms. The ability of hybrid 
robots to maneuver multiple locomotion modes increases the flexibility of the system. Research on combining both terrain and aerial locomotion capabilities in a robot is still in its beginning stage. The ability of the robot to reach the target position quickly and to avoid large terrestrial obstacles by flying expands its application in the area of search and rescue and military surveillances [7].

The advent of drone technology showed a new path for aerial robotic system. HERALD [8], HyTAQ [7], and Pens-FlyCrawl [9] are some of the pioneer robots with aerial and terrain locomotion. HERALD (Hybrid Exploration Robot for Air and Land Deployment) [8] has three robots - a quadrotor and two snake robots, which connects using a magnetic docking mechanism. The snake robots can perform wheeled locomotion individually or together with the quadrotor attached. Although the robot has three independently working robot modules, the quadrotor can fly with only one snake attached to it and both snakes are required to carry the quadrotor, which limit the usability in critical situations. HyTAQ (Hybrid Terrestrial and Aerial Quadrotor) [7] which encompasses a quadrotor. Aerial motion is achieved using the quadrotor, while the terrestrial motion is achieved by rolling the cylindrical cage using the same actuators used in the quadrotor. Rolling motion limits its payload carrying capacity. In-order to carry out terrestrial locomotion the robot have to overcome the rolling resistance. Thus, if the terrain gets a little rough the only option left is to shift to flight mode, which limits its application in catastrophe scenario. PensFlyCrawl [9] has the ability to perform aerial locomotion by using bi-copter mechanism and crawl by adding simple lever with tilting landing gear which could rotate 360 degrees. Complex controls of the bi-copter and simple terrestrial locomotion mechanism limit its use cases.

Based on the above mentioned works it is clear that there is a need for hybrid robot in search and rescue and disaster zone assistance. Thus, this research presents "Hybrid Locomotion Mobile Robot (HyLMoR)" with aerial and terrain locomotion. HyLMoR is designed considering search and rescue aspects. Most of the other robots in this category uses wheel-leg or crawling for terrain locomotion, where legged locomotion is much better compared to that in uneven terrain. In HyLMoR the modularity in components are considered where the quadruped and quadrotor modules are combined independenty. This results in independency between two modules and makes controllability simple.

\section{Factors that influence the design}

The design process of the robot involves understanding the physical problems associated with its manufacturing and should incorporate all possibilities and contingencies that preempts, so that all chances of errors are avoided. This involves producing CAD diagrams and models that gives visual description of the robot. Then analysis of the assembled model is done so as to check how it performs in real conditions. If necessary, changes are made on the design with respect to the results obtained after analysis.

Sufficient care must be taken to not be too economical at the cost surrendering the performance of the robot. Since there is a trade-off between price of the components and their performance. Hence optimised function of both has to be selected, so that our requirements are satisfied.

\subsection{Desired objectives of robot design}

The overall goal is to develop a robotic platform with aerial and terrain locomotion, that is apt for search and rescue, disaster zone assistance and surveillances. The main idea is quadrotor thrust can be used for the stability of the system in uneven surfaces and it can also be used to tackle large obstacles by flying over it. The four legs enable it to walk statically stable. In order to achieve this, the desired objectives of developing such a platform include:

- High stability: The robot must persist static stability in the absence of external forces and the gait must be planned in such a way that it makes the robot as stable as possible $[10,11]$.

- Minimum energy expenditure: The placement of the motors for quadrotor and the quadruped legs should take into consideration the energy cost optimisation. Hence selection and placing the motors will be a key design component of the robot.

- Affordable monetary cost: The motors should not be too expensive nor too cheap thus performance of motor is not compromised. Materials should be easily available in the market.

- DOF: Using a quadruped with 3 DOF leg adds omnidirectionality $[10,11]$.

- Leg configuration: Four legs with spider configuration, suitable for off-road performance have been selected for the proposed design. Spider type leg can extend its toe within a wide range not only forward and backward but also in upward and downward areas around the hip joint. This makes the robot to move even on rough environment and it requires less energy when compared with horse type $[10,11]$.

- Effects of thrust to weight ratio: The payload of the robot plays a key role while designing the quadrotor part. As the weight increases the thrust needed also increases, hence energy required also increases. So, the payload should be kept as small as possible and thrust to weight ratio as large as possible [12-14].

- Torque of joint motors: The selection of torque of the joint motor is an important criterion while designing. It is safe if the torque selected for joint-motors is larger than the required torque at each joint. This reflects in the 
movement of the robot and the static stability of the robot.

- Landing gear: In the event of a hard landing, it is the landing gear that protects the frame and other components including the joint motors. It is basically a damping mechanism which protects all the components by absorbing the vibration while landing [12-14].

\subsection{Selection of material}

The most popular materials available includes carbon fibre, aluminium, steel and titanium. Their properties are compared so as to find the right choice for each part (Table 1).

- Carbon Fibre: It has flooded the market at almost all levels, it has got an unbeatable stiffness-to-weight ratio. It is one among the most popular material.

- Aluminium: High-end aluminium is a perfect choice while making a light, stiff frame for the robot and it is available at reasonable price.

- Steel: It will last a life time. The strength and durability of steel means it is minimal fuss in maintenance.

- Titanium: This metal possesses characteristics from aluminium and steel. It has better shock absorption than that of aluminium but is not quite as light.

Table 1. Material comparison

\begin{tabular}{|c|c|c|c|}
\hline Material & $\begin{array}{c}\text { Modulus of } \\
\text { Elasticity } \\
(\mathrm{GPa})\end{array}$ & $\begin{array}{c}\text { Tensile } \\
\text { Strength } \\
(\mathrm{MPa})\end{array}$ & $\begin{array}{c}\text { Density } \\
\left(\mathrm{g} / \mathrm{cm}^{3}\right)\end{array}$ \\
\hline Carbon Fiber & 220 & 760 & 1.7 \\
\hline $\begin{array}{c}\text { Aluminium } \\
(6061-\mathrm{T} 6)\end{array}$ & 69 & 310 & 2.7 \\
\hline $\begin{array}{c}\text { Stainless Steel } \\
\text { (404) }\end{array}$ & 200 & 1790 & 7.80 \\
\hline Titanium & 116 & 434 & 4.5 \\
\hline
\end{tabular}

Each material has different qualities. Comparing material properties alone, carbon fiber will be the material of choice. However, considering the weight to strength ratio and price criteria aluminium alloy (6061T6) is selected for building up the robot.

\section{Model development and analysis}

Here, the main focus is on designing the model in the CAD software CATIA. Each part is designed individually and then they are then assembled together to configure the final design. The analysis of the robot is done in ANSYS. The modelling of this robotic platform is done in two phases, as an idea about the payload is required in order to design the quadrotor. Hence the design process includes the designing of quadruped section, followed by that of the quadrotor section.

\subsection{Design of quadruped section}

For the proposed system a terrain locomotion mechanism is required for it to move through its environment. As it is designed to be used on uneven surface, it requires high stability, thus legged locomotion is selected. The choice of static stability insists number of legs to be equal to or more than four [11]. Hence, a quadruped module is considered.

The main body frame of the quadruped consists of two aluminium sheets, each roughly of dimension $200 \mathrm{~mm}$ x $200 \mathrm{~mm}$ x $2 \mathrm{~mm}$ linked parallely using cylindrical spacers of radius $3 \mathrm{~mm}$ and length $30 \mathrm{~mm}$ as shown in Fig. 1. Further modifications to the square shape are made to reduce the weight of the base. This central body frame is used for attaching legs and other components (Fig. 1).



Fig. 1. Main-body structure

The next component to design is the leg of the robot. As explained in the design objective (Section 3.1) legs are arranged in spider configuration and each leg has got 3DOF. Hence each leg is configured by three parts, namely hip, femur and tibia (Fig. 2). The leg length which is around $260 \mathrm{~mm}$ has been choosen so as to give the optimum stride length with respect to the height of obstacles that it can traverse (here standard bricks of size $190 \mathrm{~mm} \times 90 \mathrm{~mm} \times 90 \mathrm{~mm}$ are considered as obsatacle). Initially link lengths are selected in such a way that ratio of link lengths (Femur : Tibia) is around 0.42 [15]. After considering the mechanical constrains, the link lengths are slightly modified. The link lengths thus chosen are shown Table 2.

Table 2. Link lengths

\begin{tabular}{|c|c|c|c|}
\hline Link & Hip & Femur & Tibia \\
\hline Length $(\mathrm{m})$ & 0.063 & 0.082 & 0.1536 \\
\hline
\end{tabular}

The hip link which is the first link from the main body which is also known as coxa. This part makes link between the servo attached to the main-body and servo attached to femur. This is just a small link close to the main body. The femur link is the middle segment of leg. The dimensions have been kept such that the linear distance between the joints amounts to $82 \mathrm{~mm}$ whereas the total link is $96 \mathrm{~mm}$. The last segment of leg that contacts the ground called as tibia. The walking traction 
is bettered by attaching a rubber cap at the end of tibia. As explained in the design objective a landing gear has to be provided so as to avoid damage on the robot while hard landing occurs during the transition from aerial mode to terrain mode. In order to tackle it a simple helical spring mechanism is added to the tibia part that provides damping.



Fig. 2. Assembled leg

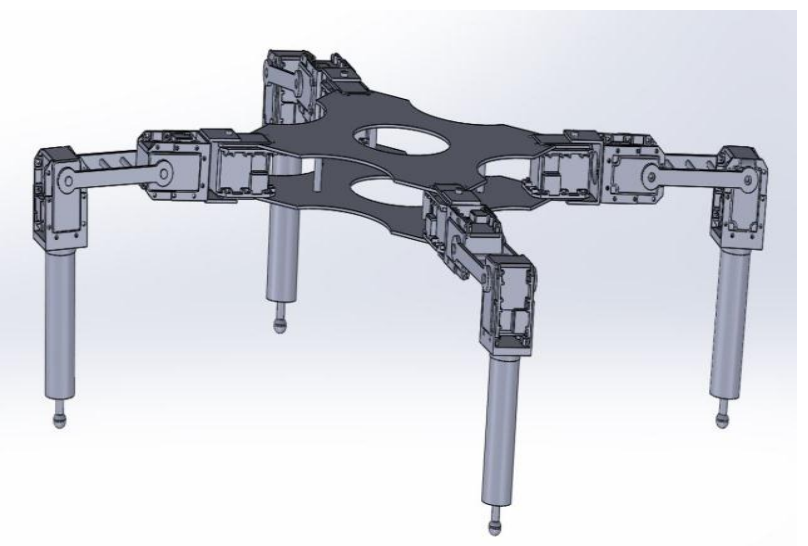

Fig. 3. Assembled quadruped section

Assembling of different parts involves joining the hip, femur and tibia to form the leg (Fig. 2), subsequently followed by joining all these four legs with the main chassis to build up the quadruped section (Fig. $3)$.

\subsection{Analysis of quadruped section}

Static state finite element method (FEM) is used to investigate various forces and deformation of each part of the robot. The deformations, stress and strain on the structure of robot is verified by static structural analysis.

\subsubsection{Static structural analysis}

Structural analysis is a key part of the design process. In this analysis, the various supports and load acting under gravity on the robot are considered. It also gives an idea whether the system design needs any changes.
Here, gravitational force has been applied and point supports has been given at the end of tibia part in all the four legs. Mass of quadrotor section and electronic components are applied on top plate of main chassis.

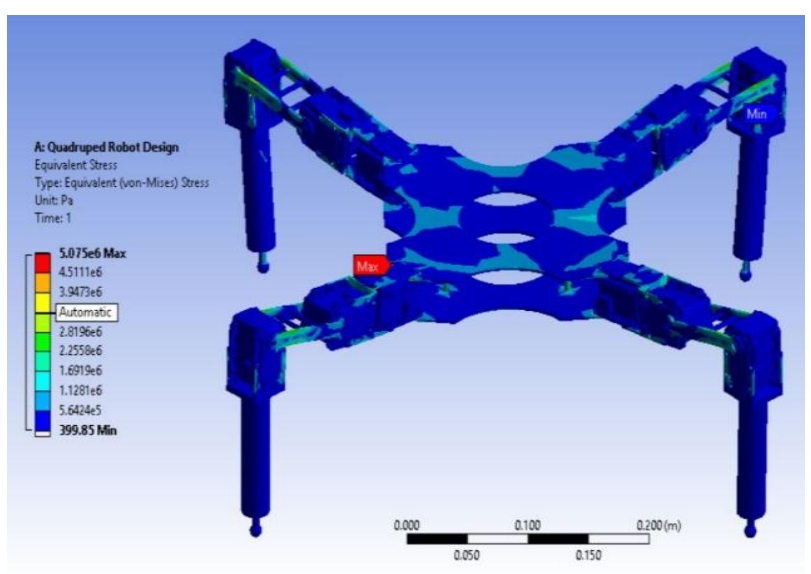

Fig. 4. Stress distribution contour of quadruped section

The resulting stress distribution contour of quadruped section is shown in Fig. 4. The maximum stress of $5.075 \times 10^{6} \mathrm{~Pa}$ is found at main body. The system is made of aluminum alloy (6061-T6) with a yield stress of $2.7 \times 10^{8} \mathrm{~Pa}$, hence the stress is in allowable range.

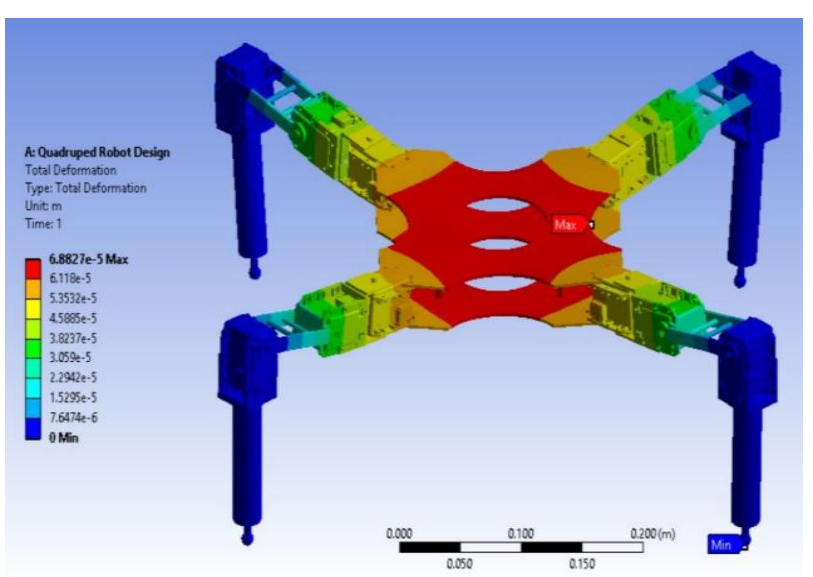

Fig. 5. Deformation contour of quadruped section

The resulting deformation contour of quadruped section is shown in Fig. 5. The maximum deformation that occurs at the main body is just $6.8827 \times 10^{-5} \mathrm{~m}$ which is smaller than a millimetre, hence the deformation does not make any impact on model.

\subsection{Design of quadrotor section}

The payload of the robot plays a key role while designing the quadrotor part. It is because of this reason the quadruped section was designed first, so that an idea about the mass of the robot is obtained.

A quadrotor has four arms, each connected to one motor. The arms are symmetric about two axis. First the payload of the quadrotor has to be calculated [12]. The total mass of the robot is denoted by $M_{T}$ and is given by 


$$
M_{T}=\left(M_{Q P}+M_{Q R}+M_{E P}\right)
$$

where $M_{Q P}$ represents the mass of quadruped section, $M_{Q R}$ the mass quadrotor section and $M_{E P}$ the mass of electronic parts. If $T_{Q}$ is the thrust required by quadrotor then

$$
T_{Q}=M_{T} \times g
$$

where $g$ represents the gravitational acceleration. While designing a quadrotor, its components are designed to lift double the payload [7]. Thus, thrust considered while designing is denoted by $T_{D}$ and is given by

$$
T_{D}=2 \times T_{Q}
$$

Since it is a symmetric design, quarter of quadrotor is to be analysed. Thus, the thrust of a single propeller $\left(T_{S P}\right)$ is given by

$$
T_{S P}=\left(T_{D} / 4\right)
$$

The propeller has to be selected such that it is capable of producing the required thrust. On the basis of calculations done, each propeller should produce $15 \mathrm{~N}$ thrust (Eq. 4). Various standard propellers have been analysed using Computational Fluid Dynamics (CFD) analysis (explained later in Section 4.4.1). From the results of the CFD analysis it is found that a $0.254 \mathrm{x}$ $0.119 \mathrm{~m}(10 \times 4.7 \mathrm{inch})$ propeller is capable of producing the required thrust.

Quadrotor uses two sets of identical fixed pitched propellers i.e. two counter clockwise (CCW) propellers and two clockwise $(\mathrm{CW})$ propellers. Variation of RPM of these propellers is used to control lift and torque of the quadrotor. The arm of the quardrotor is designed such that, it withstands a maximum thrust of $15 \mathrm{~N}$. The dimensions have been kept such that the linear distance between the edge and centre point of motor attachment slot to $232 \mathrm{~mm}$ whereas the total link is $246 \mathrm{~mm}$. The link length is calculated considering that propeller should stand out of main chassis.

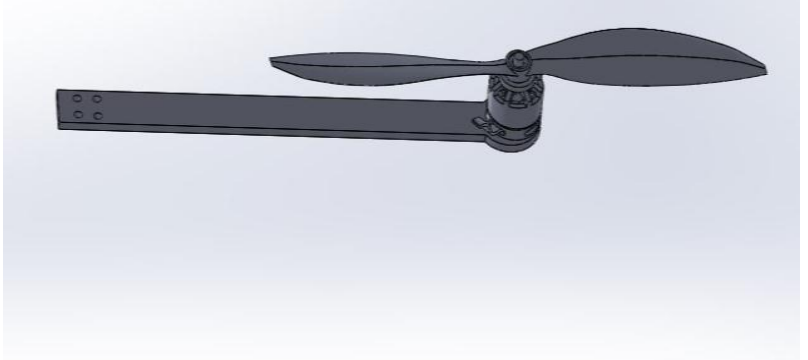

Fig. 6. Quadrotor arm assembled with $\mathrm{CCW}$ propeller

Different parts of the quadrotor arm (joining arm, brushless DC motor and propeller) are assembled as shown in Fig. 6.

\subsection{Analysis of quadrotor section}

CFD is used for estimating propeller characteristics flow region. Here CFD analysis was carried out to find the output thrust produced by the propeller. Static structural analysis has been performed to analyse the various forces and deformations acting at various parts of quadrotor arm.

\subsubsection{CFD analysis}

As explained in the discussion on designing the quadrotor section (Section 4.3), each propeller of the quadrotor should be able to produce a torque of $15 \mathrm{~N}$. So it is required to validate the performance of the propeller using fluid flow simulation. Various standard propellers are subjected to CFD analysis and it is found that $0.254 \mathrm{x}$ $0.119 \mathrm{~m}(10 \times 4.7 \mathrm{inch})$ is capable of producing the required thrust at RPM less than the optimal RPM (three-fourths of maximum RPM of BLDC motor considered i.e. $1571 \mathrm{rad} / \mathrm{s})$.

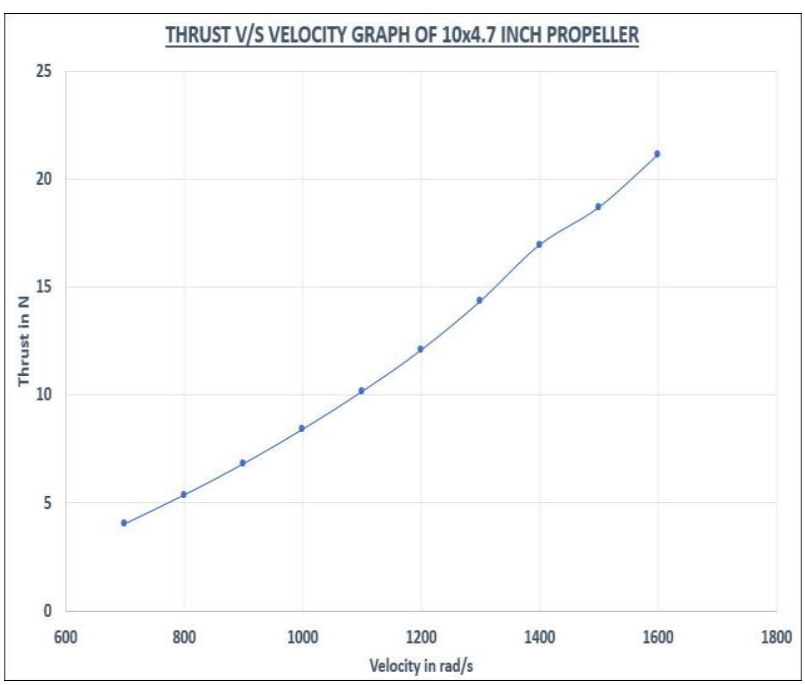

Fig. 7. Thrust v/s Velocity graph of $10 \times 4$.7inch propeller

Fig. 7 shows the various thrust values obtained from CFD analysis while $0.254 \times 0.119 \mathrm{~m}(10 \times 4.7$ inch $)$ propeller is simulated at different velocity. It is clear from the graph that $15.03 \mathrm{~N}$ thrust has been obtained from the propeller at $1350 \mathrm{rad} / \mathrm{s} \quad(12891.55 \mathrm{rpm})$. Therefore, this propeller is selected.

\subsubsection{Static structural analysis}

The arm of the quadrotor section should be designed to withstand a force of $15 \mathrm{~N}$, which is the maximum thrust value used for the flight. Because of the symmetry of the quadrotor section, single quarter arm is required to be analysed. While simulating gravitational force has been applied, fixed supports has been the edge where holes are provided for fixture (left-side of Fig. 8). A force of $15 \mathrm{~N}$ is applied in upward direction where propeller will be placed (right-side of Fig. 8). 




Fig. 8. Stress distribution contour of quadrotor arm

The resulting stress distribution contour of quadrotor arm, while subjected to the force of $15 \mathrm{~N}$ is shown in Fig. 8. The maximum stress of $3.88 \times 10^{7} \mathrm{~Pa}$ is found at arm. Since the frame is made of aluminum alloy (6061-T6) with a yield stress of $2.7 \times 10^{8} \mathrm{~Pa}$, the stress is in allowable range.

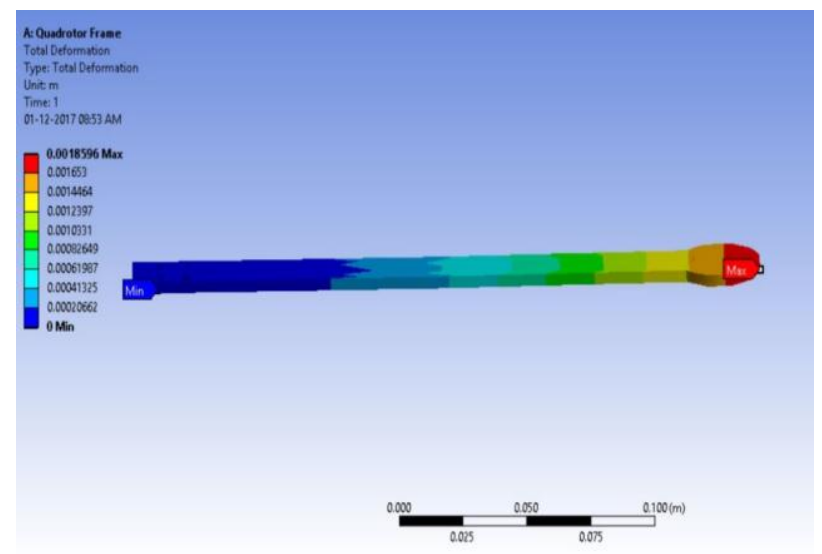

Fig. 9. Deformation contour of quadrotor arm

The deformation contour of the quadrotor arm when subjected to the $15 \mathrm{~N}$ force is shown in Fig. 9. The maximum deformation that occurs at the main body is just $0.0018 \mathrm{~m}$ which is very small, hence the deformation doesn't make any impact on model.

\subsection{Development and assembly of HyLMoR}

Both quadruped and quadrotor section parts designed are assembled together, such that robot capable of aerial and terrain locomotion is obtained.

The dimensions of the base plate for quadrotor frame has been assumed to be $142.4 \mathrm{~mm} \times 142.4 \mathrm{~mm} \times$ $2 \mathrm{~mm}$. It is attached to the main chassis by cylindrical spacer of radius $3 \mathrm{~mm}$ and length $60 \mathrm{~mm}$ (Fig. 10). Modification in the square shape is made in order to reduce the weight of the base and fillet of $2 \mathrm{~mm}$ is applied on all the sharp corners inorder to avoid the stress concentration.



Fig. 10. Main chassis

All the parts designed in the quadruped section and quadrotor sections are assembled together. Thus the final robot assembly is completed (Fig. 11).

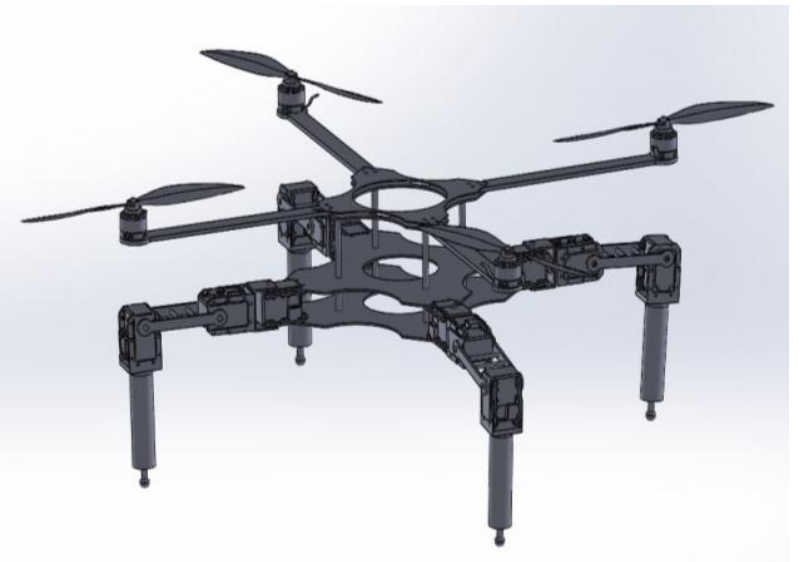

Fig. 11. Full model assembly (HyLMoR)

\subsection{Analysis of HyLMoR}

Static analysis was done to analyse the various forces and deformation of each part of the robot. Drop test was done to determine the critical landing velocity of the robot.

\subsubsection{Static structural analysis}

Each arm of the quadrotor section should be designed to withstand a force of $15 \mathrm{~N}$, which is the maximum thrust value used of the flight. The main body should be able to carry various electronic components (battery, control board etc.), so mass of electronic components are also considered. Gravitational force has been considered and support forces are applied at the tibia part. 


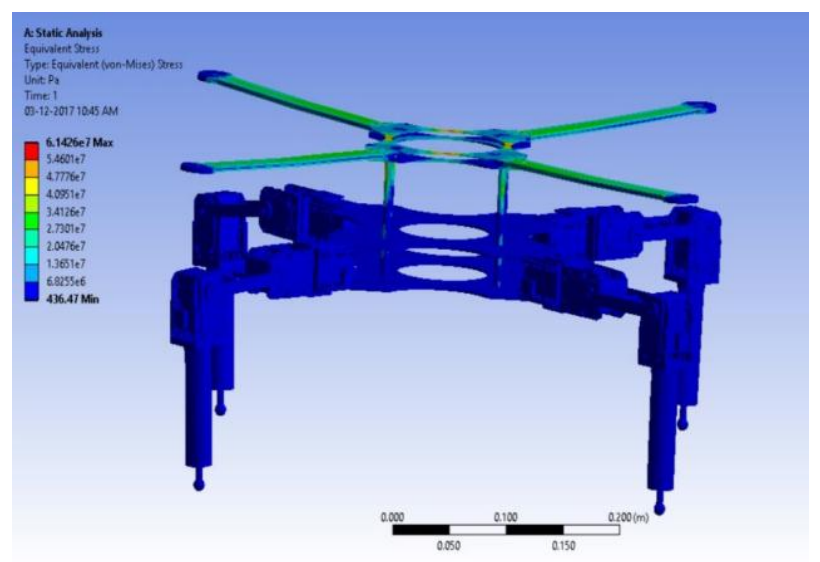

Fig. 12. Stress distribution contour of HyLMoR

The resulting stress distribution contour of HyLMoR is shown in Fig. 12. The maximum stress of $6.142 \mathrm{x}$ $10^{7} \mathrm{~Pa}$ is found at body plate that supports the quadrotor arms. Since the frame is made of aluminum alloy (6061T6) with a yield stress of $2.7 \times 10^{8} \mathrm{~Pa}$, the stress is in allowable range.

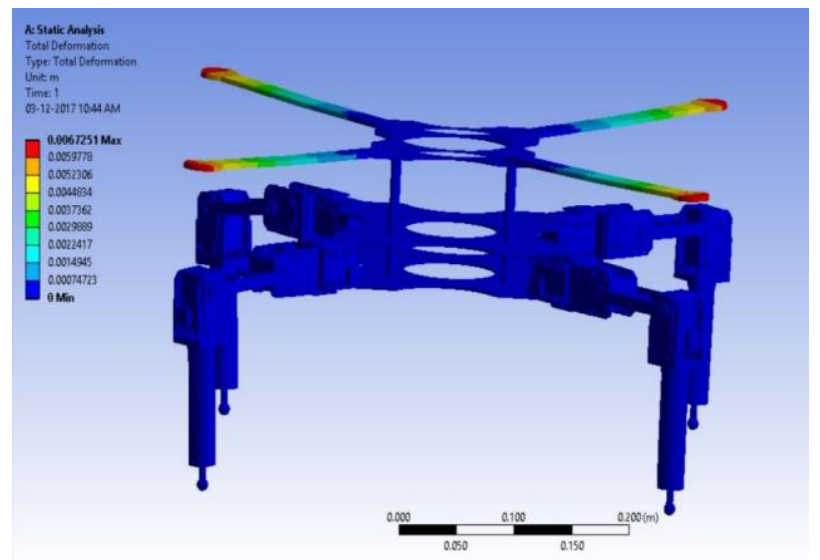

Fig. 13. Deformation contour of HyLMoR

The resulting deformation contour of HyLMoR is shown in Fig. 13. The maximum deformation that occurs at the main body is just $0.0067 \mathrm{~m}$ which is acceptable, hence the deformation doesn't make an impact on model.

\subsubsection{Drop test analysis}

The landing gear of the robot is analysed to understand the critical falling velocity of the robot design. For this, simulation of the impact on the landing gear for several velocities is performed. While analysing, gravitational force and the force at the tibia part are considered. In the simulations, the landing surface is assumed to be made of concrete.

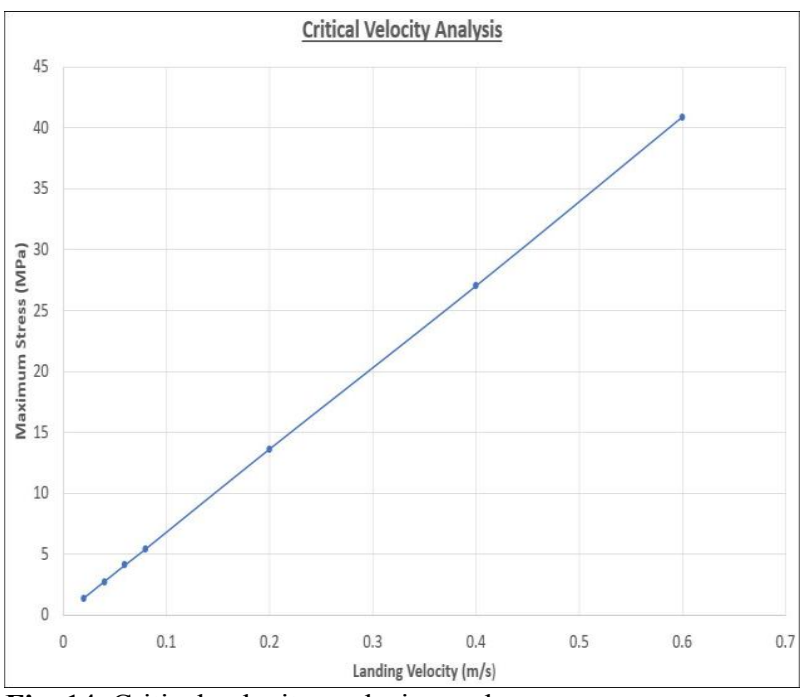

Fig. 14. Critical velocity analysis graph

Eventhough the maximum yield stress of aluminium alloy (6061-T6) is $2.7 \times 10^{8} \mathrm{~Pa}$, the stress of the material used for making the servo motors at the joints of the leg also has to be cosidered. Acrylonitrile butadiene styrene (ABS) with a tensile stress of $4.4 \times 10^{7} \mathrm{~Pa}$ is used for making the casing of the motor. Therefore, from Fig. 14 landing velocity which produces a maximum stress below $30 \mathrm{MPa}$ is considered, thus the critial velocity is kept at $0.4 \mathrm{~m} / \mathrm{s}$ to ensure no damage occurs to robot. This is with the assumption that the quadrotor would produce a drag reducing the velocity.

\section{Conclusion}

The primary motivation of this research has been to design a hybrid mobile robot with aerial and terrain locomotion capability. Thus, the robot can be used for search and rescue, disaster zones assistance and surveillances.

In this project, a robot with a combination of 12 DOF quadruped and quadrotor was designed. CAD models were made and fine-tuned by doing various simulation analysis. The final design is a compromise betweeen specification required for the robot, expenditure and overall weight of the robot. It's found that required thrust of $15 \mathrm{~N}$ can be made using CFD analysis (Section 4.4.1). A maximum of $0.4 \mathrm{~m} / \mathrm{s}$ is obtained as the critical velocity for landing from drop test analysis (Section 4.6.2). From the static analysis, with a maximum stress of $6.142 \times 107 \mathrm{~Pa}$ (Section 4.6.1) and maximum deformation of $0.0067 \mathrm{~m}$ (Section 4.6.1). The various analysis results did not exceed the safety limits, hence the robot design made is safe and reliable.

It is clear that a final validation is only possible after evaluation of the constructed model. However, the simulation studies lay the foundation for building the robot capable of aerial and terrestrial locomotion. 


\section{References}

1. Y. Ichikawa, N. Ozaki, and K. Sadakane, IEEE Trans. on Systems, Man, and Cybernetics, SMC-13, pp. 1089-1093, (1983).

2. Mobile rescue robots, https://www.nccr robotics.ch/research-areas/mobile-rescuerobots/research-overview-mobile-rescue-robots/, (2017-12-05).

3. F. Seljanko, IEEE Int. Conf. on Mechatronics and Automation, ICMA, (2012)

4. P. Ben-Tzvi, A. A. Goldenberg and J. W. Zu, IEEE Int. Conf. on Robotics and Automation, Pasadena, pp. 2307-2312, (2008).

5. T. Fujita, T. Sasaki and Y. Tsuchiya, IEEE Int. Symp. on Safety, Security, and Rescue Robotics (SSRR), West Lafayette, IN, pp. 1-6, (2015).

6. P. Ratsamee et al., IEEE Int. Symp. on Safety, Security, and Rescue Robotics (SSRR), Lausanne, pp. 62-67, (2016).

7. A. Kalantari and M. Spenko, Robot.Autom.(ICRA), 2013 IEEE Int. Conf., pp. 4445-4450, (2013).

8. S. Latscha, M. Kofron, A. Stroffolino, L. Davis, G. Merritt, M. Piccoli, and M. Yim, IEEE/RSJ Int. Conf. on Intelligent Robots and Systems, pp. 18681873, (2014).

9. S. Kuswadi, M. N. Tamara, D. A. Sahanas, G. I. Islami, and S. Nugroho, Int. Conf. on Knowledge Creation and Intelligent Computing (KCIC), pp. 80-87, (2016).

10. S. Hirose, , 3rd CISM-IFToMM Int. Symp. on Theory and Practice of Robots and Manipulators, pp. 357-375, (1978).

11. D. C. Kar, J. Robotic Syst., pp. 671-686, (2003).

12. M. Muflikhun, E. Magsino, and A. Y Chua, , RCMME-2014, (2014).

13. D. Singla, Design of a Quad Rotor, https://sites.google.com/site/designofaquadrotorgrou p28/, (2017-12-05).

14. C. Benson, How to Make a UAV / Drone, https://www.robotshop.com/blog/en/makeuavlesson-2-platform-14448, (2017-12-05).

15. M. Silva, 14th Int. Conf. on cimbing and walking robots and the support technologies for mobile machines, France, (2011). 